\title{
Experiencias
}

\section{¿Atención Temprana a distancia? Experiencia en la covid-19 (marzo-julio 2020) y reflexiones para el futuro}

Remote early care? Covid-19-mediated experience (March to July 2020) and considerations for the future

\author{
P. Martín Andrade
}

\section{Resumen}

Se expone en este artículo el cambio en el modelo de trabajo que se hubo de llevar a cabo en el servicio de Atención Temprana (AT) del Centro de Recursos Educativos (CRE) de la ONCE en Madrid durante la situación de estado de alarma originada por la pandemia provocada por la covid-19. Partiendo de la definición de AT y de algunos de sus principios inspiradores, así como de la descripción de cómo se venía haciendo el trabajo hasta ese momento, se explica la adaptación que hubo de hacer el equipo en su dinámica interna ante la nueva situación; así como, en el trabajo directo, aunque a distancia, que se realizó por parte de los profesionales con las familias de los niños, haciéndose una valoración positiva de la misma, a tenor de las circunstancias. En base a esta experiencia de atención a distancia (telemática) se hace una reflexión sobre cuáles son las condiciones para que se pueda plantear su viabilidad en el ámbito de la AT en situaciones que no son de emergencia social. Se llega a la conclusión de que, con las condiciones adecuadas, esta modalidad de atención puede no reducirse a situaciones excepcionales; aunque en ningún caso, puede ser utilizada para reemplazar la atención presencial.

\section{Palabras clave}

Atención Temprana. Atención a distancia. Atención telemática. covid-19. 


\begin{abstract}
This article describes the changes that had to be made in the working model for the early care services provided by ONCE's educational resource centre at Madrid during the state of emergency called to deal with the covid-19 pandemic. The discussion builds on the definition of early care, its underlying principles and the description of how the service was being provided prior to the crisis. The focus is on the adaptations that had to be made in team internal dynamics under the new circumstances and in the direct albeit remote work performed by professionals with children's families. The overall assessment is favourable, in light of the situation prevailing. Based on this remote care experience, the author reflects on the conditions that would contribute to its viability for early care in non-emergency situations. The conclusion reached is that although this type of care need not be confined only to emergencies, it should not under any circumstances be deemed a replacement for in-person care.
\end{abstract}

\title{
Key words
}

Early care. Remote care. ICT-mediated care. Covid-19.

\section{Introducción. Nuestro modelo de atención}

La pandemia de la covid-19 está teniendo un impacto significativo en muchos aspectos de la sociedad, lo que se ha plasmado en nuestro quehacer cotidiano. En concreto, en el marco del trabajo en un servicio de AT, como es el del CRE de la ONCE en Madrid, una vez que en el pasado mes de marzo fue necesario suspender la atención presencial. Pronto se vio la necesidad de poder garantizar a los niños con discapacidad visual algún tipo de atención que permitiese cumplir con los objetivos previstos y seguir avanzando, como siempre, con nuevos retos.

Afortunadamente, la AT se reconoce como un derecho al que tienen todas las personas que presentan diversidad funcional; en nuestro caso, visual.

Para centrar el tema, tomamos como definición de AT la que nos ofrece el Libro blanco de la Atención Temprana (Federación Estatal de Asociaciones de Profesionales de Atención Temprana, 2005, p. 12): 
Se entiende por Atención Temprana el conjunto de intervenciones, dirigidas a la población infantil de 0-6 años, a la familia y al entorno, que tienen por objetivo dar respuesta lo más pronto posible a las necesidades transitorias o permanentes que presentan los niños con trastornos en su desarrollo o que tienen el riesgo de padecerlos. Estas intervenciones, que deben considerar la globalidad del niño, han de ser planificadas por un equipo de profesionales de orientación interdisciplinar o transdisciplinar.

Es necesario que destaquemos aquí algunos de los principios en los que se inspira el modelo de AT de la ONCE:

- La consideración del déficit visual como factor de vulnerabilidad. Este implica una importante limitación en la percepción e interpretación de estímulos.

- Carácter preventivo. Una intervención adecuada y a tiempo incide directamente en una mejor disposición de recursos personales por parte del niño y su familia para afrontar dificultades en el futuro, y puede evitar posteriores intervenciones más difíciles y más costosas.

- Carácter globalizador como forma de entender al niño. El conjunto de la intervención se dirige a favorecer el desarrollo global del menor, para lo cual es preciso contemplar el conjunto de factores que pueden incidir en dicho desarrollo.

- Enfoque habilitador. Proporcionar al niño experiencias que le resulten motivantes, y pueda, a través de ellas, descubrir, explorar y conocer el mundo que le rodea.

- Coordinación interdisciplinar. La atención que se presta debe ser el resultado de la mirada compartida del conjunto de profesionales que intervienen en cada caso y con cada familia.

- Complementariedad. Con carácter general, la atención prestada por los equipos específicos a los niños con discapacidad visual debe ser complementaria a la que reciban por parte de otros profesionales o centros en los que sean atendidos.

Por tanto, la intervención en AT se ha de dirigir, de forma prioritaria, a atender el desarrollo emocional y el proceso de apego con las figuras de cuidados. En paralelo, se trabajan los aspectos esenciales del desarrollo en los que la discapacidad visual pueda estar interfiriendo, así como su autonomía y socialización. 
Para llevar a efecto el trabajo de AT en el caso de Madrid, se utiliza prioritariamente la sede del equipo, ubicada en el CRE de la ONCE, si bien en los casos de niños con inmunodeficiencias se les atiende en su domicilio y en aquellos que concurren otras deficiencias junto a la visual se les realiza un seguimiento mensual en los Centros públicos o concertados de Atención Temprana (CAT) que tiene la Comunidad de Madrid y que dependen de la Consejería de Servicios Sociales. También se realiza este seguimiento en los centros privados de Atención Temprana, cuando los niños no asisten a los primeros, así como, en los hospitales, cuando las circunstancias lo requieren.

En este momento, nuestro equipo no atiende a los niños en las escuelas infantiles ni en los colegios, competencia del Equipo de Orientación Educativa y Psicopedagógica (EOEP) Específico de Discapacidad Visual, en acuerdo con la ONCE, con el que procuramos coordinarnos, pues no son pocos los niños que son atendidos por ambos equipos.

Las sesiones de atención a los menores de 3 años son de carácter individual (niño y familia), con una frecuencia semanal en la mayoría de los casos.

Con los niños mayores de 3 años que, mayoritariamente, se encuentran escolarizados, son atendidos en sesiones individuales por nuestro equipo cuando se estima la necesidad. Además, a los niños de educación infantil entre 3 y 6 años se les ofrece asistir a sesiones grupales, en forma de talleres, al objeto de favorecer el proceso de ajuste personal y la socialización con otros niños con discapacidades similares. Estas sesiones se realizan una vez en semana, durante dos horas, en horario de tarde. En paralelo, se lleva a cabo una Escuela de Padres, dirigida a los progenitores de niños de este grupo de edad.

Todas estas prácticas tuvieron que ser canceladas en el momento en que, como consecuencia de la pandemia, se declaró el estado de alarma y, acorde con la situación, el confinamiento domiciliario, por lo que nos vimos obligados a un cambio en el modelo de atención a esta población.

\section{El reto de la atención a distancia}

El cambio en el modelo de atención ha supuesto un desafío. No se trataba solo de contactar con unas familias ya de por sí muy preocupadas por la incertidumbre en el ámbito de la salud, en algunos casos del laboral y, en todos, por lo que la situación 
podría afectar al bienestar y desarrollo de su hijo. Era necesario, si se quería mantener algún criterio de calidad, poder evaluar la situación de los niños y comunicarse eficazmente con sus padres, dado que, más que nunca, debían asumir el papel de intermediarios entre nosotros y su hijo. En esta situación no cabía la posibilidad del «modelado» - práctica por la que los padres imitan la acción del profesional-, al que ellos y nosotros estamos acostumbrados cuando se quiere favorecer la comunicación y alcanzar nuevos logros.

El equipo de AT del CRE de Madrid cuenta con una vasta experiencia; sin embargo, no resultó fácil adaptarla a las nuevas circunstancias. Como profesionales, más que nunca hemos sentido necesario tener encuentros (virtuales) entre los compañeros, compartir dudas y experiencias, conocer las soluciones encontradas para situaciones concretas; en definitiva, sentir que no era un problema individual sino del grupo ha facilitado la tarea. Sin embargo, además de nuestra falta de experiencia, carecíamos de referencias respecto a los posibles resultados que, con esta metodología, se podrían obtener con relación al desarrollo de los niños y el ajuste emocional con sus familias. Agravándose la situación por el hecho de que, a algunos miembros del equipo, en concreto la psicóloga y la trabajadora social, se las incluyó en un expediente de regulación temporal de empleo (ERTE) a tiempo completo durante esos meses, por lo que, entre otras consecuencias, no pudieron participar de la dinámica, quedando activos solo los cuatro maestros.

Como ya se ha dicho, lo que teníamos todos claro al ser suspendida la atención presencial en nuestro servicio era la necesidad de continuar con la atención a las familias, $y$, a través de ellas, a sus hijos. El recurso principal eran los medios telemáticos de los que cada uno dispusiese (en ese momento no era de recibo pedir a las instituciones una dotación ad hoc para cada cual). Por lo tanto, los profesionales pusimos a disposición nuestros medios, muy variados: en algún caso un ordenador con Windows XP y correo electrónico particular Gmail, más un teléfono móvil básico; eso sí, con WhatsApp. Lo que resultó suficiente, pues tampoco ninguna familia sugirió conectarse por medios muy sofisticados, pero tampoco hubo ninguna que no pudiese conectarse, aunque fuera por vía telefónica.

Para poder proseguir con la atención a los niños fue necesario hacer un acercamiento a la situación en que se encontraban las familias. Los padres, en este contexto, han asumido roles simultáneos, el propio de padres y el de maestros; en muchos casos, compatibilizándolo con el trabajo dentro o fuera de la casa. Y siempre, como queda dicho, con incertidumbre.

Martín, P. (2021). ¿Atención Temprana a distancia? Experiencia en la covid-19 (marzo-julio 2020) y reflexiones para el futuro. RED Visual: Revista Especializada en Discapacidad Visual, 77, 172-181. https://doi.org/10.53094/ IKKR7253. 
Todos, ellos y nosotros, debimos asumir nuevas rutinas en nuestras vidas. Algunas familias al principio lo tomaron como algo «temporal», por lo que relajaron sus costumbres; pero pronto comenzaron a agobiarse, pues veían que sus hijos podían verse perjudicados por la falta de atención, ya que el tiempo pasaba y no se veía el final. Los profesionales también nos sentíamos inseguros en esta situación; aunque paulatinamente, al tratarse de familias con las que ya habíamos establecido unos vínculos, fue posible obtener información concreta de la situación en la casa y acordar los momentos en que estableceríamos los contactos. No se trataba de interesarse informalmente por el niño y la familia, sino de poder continuar con el trabajo que se había venido realizando, regularizando para ello unas sesiones (vía telemática) en las que poder hablar sobre las adquisiciones del hijo y seguir su desarrollo, ajustando el programa en función de las circunstancias.

En la medida en que se ha podido establecer con carácter regular la comunicación con una familia, ha sido posible obtener una mayor información de su situación y de cómo estaba afectando esta a lo que es el principal objeto de nuestro trabajo: ayudarlas con nuestro apoyo a favorecer el desarrollo de su hijo con discapacidad visual.

Este nuevo contexto nos ha llevado a interesarnos, a modo de ejemplo, en cómo estaban afrontando la situación con los hermanos mayores, pues, en bastantes casos, los padres se han visto desbordados al tratar de ayudar a sus hijos con las actividades que se les proponían telemáticamente. Unas veces por resultar difícil compatibilizar el propio trabajo con la ayuda imprescindible que el hijo precisaba desde el punto de vista técnico o para seguir las clases sin distracciones. A lo que hay que añadir la presión de las continuas referencias a la «necesaria» colaboración de la familia por parte de los maestros, que, careciendo de competencia con la nueva didáctica, en muchos casos multiplicaron las tareas encomendadas a los alumnos (y a sus padres). Sin embargo, se ha visto cómo las familias querían hablar al respecto y cómo aplicaban soluciones satisfactorias en las que se incluía también al hermano menor con discapacidad visual. En concreto, cuatro de las familias que atendí se encontraron en esta situación. Niños atendidos por mí entre los 18 meses y los 3 años, con hermanos que se llevaban con ellos entre dos y tres años. Algunas aprovechaban las actividades escolares para, adaptadas, proponérselas a ellos; en otros, el tiempo de tareas por parte de los mayores fue el momento, también para los pequeños, de hacer sus propias «tareas».

En las conversaciones con los padres, sobre todo con los de mayor edad, se hablaba de las rutinas establecidas para ese momento, lo que daba lugar, en muchos casos,

Martín, P. (2021). ¿Atención Temprana a distancia? Experiencia en la covid-19 (marzo-julio 2020) y reflexiones para el futuro. RED Visual: Revista Especializada en Discapacidad Visual, 77, 172-181. https://doi.org/10.53094/ IKKR7253. 
a poder proponer nuevos aprendizajes en el contexto de las actividades propias de la casa, como dar las pinzas para colgar la ropa, llevar el pan a la mesa, emparejar calcetines..., lo que indudablemente las podía hacer más funcionales.

Casi todas las familias enviaron vídeos con las «gracias» y los logros de los pequeños. Resultaron de utilidad, en la mayoría de los casos, para comentar con ellas lo que se apreciaba y hacerles nuevas propuestas. Pero también hay que decir que, en algún caso, no resultaron significativos, siendo difícil valorar en ellos la situación real, por lo que no sirvieron para seguir avanzando significativamente en el programa.

Como puede observarse, a lo largo de este artículo solo se hace referencia a la continuación del trabajo que ya se venía realizando con los niños que estaban siendo atendidos, hasta el momento del inicio del estado de alarma por parte de los maestros. Durante el tiempo que duró la misma no se hicieron evaluaciones de nuevos casos, pues, además de la imposibilidad de citar en la sede, tampoco se contempló la posibilidad de que la psicóloga y la trabajadora social teletrabajasen. No obstante, llegaron algunos casos derivados desde el Departamento de Afiliación de la Delegación Territorial de la ONCE a los que también se trató de dar respuesta. En estos casos la dificultad fue mayor, dado que no existía el conocimiento y la confianza que otorga la relación más directa previa. Especial dificultad se dio con algún pequeño ciego, donde resulta más relevante la labor de «modelado» por parte del profesional hacia la familia. Algo parecido ocurrió en los casos de niños con discapacidad múltiple, quienes, al no poder ser atendidos en los CAT, se hubo de realizar una labor más activa en cuanto a ofrecer pautas de actuación. En ambos casos fue muy importante poder escuchar a los padres y ofrecerles algo de contención emocional.

\section{Regreso al trabajo presencial}

En septiembre se volvió, tanto en Madrid como en la mayoría de las comunidades autónomas españolas, al trabajo presencial. En nuestro caso, ello significaba recibir a los pequeños en la sede y realizar, en la mayoría de los casos, el seguimiento presencial en los Centros de Atención Temprana de los niños con discapacidad múltiple en ellos atendidos.

No se han podido reanudar las actividades grupales que continúan haciéndose a través de plataformas en Internet. Como cabe suponer, las que se hacían con los niños

Martín, P. (2021). ¿Atención Temprana a distancia? Experiencia en la covid-19 (marzo-julio 2020) y reflexiones para el futuro. RED Visual: Revista Especializada en Discapacidad Visual, 77, 172-181. https:/doi.org/10.53094/ IKKR7253. 
de $2 .^{\circ}$ ciclo de educación infantil no se han podido hacer, siendo sustituidas por otras que, aunque dirigidas a ellos, se han hecho con sus padres. En concreto, les hemos ofrecido dos actividades, dedicando a cada una dos sesiones, con los títulos de Juegos y juguetes e ¿Y si leemos un cuento?, con las que hemos buscado sobre todo favorecer las relaciones intrafamiliares. Con la primera también hemos buscado ofrecerles recursos para favorecer la relación con otros niños dentro y fuera de la casa.

La valoración que hemos hecho los compañeros de cómo hemos encontrado a los niños después de estos meses, compartida incluso con los componentes del Seminario de Atención Temprana de la zona, es que ha sido diversa. En algunos casos, para la familia ha sido una oportunidad de poder estar con su hijo y realmente avanzar juntos; en otros, sin embargo, se ha observado un estancamiento en el desarrollo esperado. Desde luego, las condiciones y circunstancias que han rodeado esta excepcional situación no han sido las mismas para todos y, reconozcámoslo, hemos tenido que improvisar.

Como profesionales de la Atención Temprana puede valorarse positivamente esta experiencia de teletrabajo con las familias. Se ha de destacar el haber podido estar cerca de su entorno natural, la casa, aunque fuera a distancia; como correspondía en ese momento.

\section{Mirando al futuro}

Toca ahora reflexionar sobre lo hecho y mirar al futuro. Está claro que se ha vivido una situación excepcional que todos deseamos que no se repita, pero para la que es necesario estar preparados; también nos ha dejado una experiencia y algunas enseñanzas.

Este tipo de atención telemática puede aplicarse aun sin la necesidad de encontrarse en una situación excepcional. Sabiendo que nunca puede darse de forma exclusiva - ni siquiera preferente-, pero que podría ser combinada con la presencial en algunos casos y en algún momento. La atención telemática puntual puede ser un modo complementario de atención en nuestro ámbito de actuación; eso sí, para que tenga valor y sea eficaz esa conexión telemática, es imprescindible definir el objetivo que se quiere cubrir de esa manera. Así mismo, es necesario que se cumplan algunas condiciones que, si bien su falta se pudo justificar en aquel momento, de futuro sería imperdonable no subsanarlas:

Martín, P. (2021). ¿Atención Temprana a distancia? Experiencia en la covid-19 (marzo-julio 2020) y reflexiones para el futuro. RED Visual: Revista Especializada en Discapacidad Visual, 77, 172-181. https://doi.org/10.53094/ IKKR7253. 
- La práctica de la atención telemática requiere tener formación y entrenamiento específico en «técnicas de atención a distancia»: no está preparado cualquiera para llevarla a cabo.

- Se precisa también formación en los aspectos técnicos e informáticos en función de la realidad a atender: se ha de contar con los equipos adecuados y una buena conectividad.

- Se han de garantizar los aspectos de seguridad y confidencialidad de los datos, por lo que han de establecerse claramente los canales para las conexiones con las familias.

- Se han de aplicar, en definitiva, criterios de calidad en su utilización.

En esta reflexión sobre la atención telemática es necesario incidir también en su efecto sobre la propia dinámica del equipo de AT. En este sentido, ha resultado evidente que es posible reunirse de forma remota y trabajar sobre determinados temas, siempre planificando los tiempos para ello. Ya se ha destacado la importancia que ha tenido la comunicación entre los miembros del equipo durante la pandemia, que se tradujo en una mejora de las condiciones de trabajo y, sin duda, redundó positivamente en el mismo. Quizá también puedan utilizarse las nuevas formas de utilizar la tecnología para promover la evaluación, la observación y el seguimiento del progreso en determinados casos, al poderse compartir vídeos. Así mismo, en su caso, podría servir de autoevaluación para el profesional y de análisis de su actuación con los miembros del equipo. Todo esto supone un reto, pues es preciso cambiar el esquema de trabajo y la responsabilidad sobre el mismo.

\section{Conclusión}

Con las condiciones adecuadas, esta modalidad de atención puede no reducirse a situaciones de emergencia, aunque, en ningún caso, puede ser utilizada para reemplazar la atención presencial. El uso de la tecnología para realizar visitas domiciliarias puede ahorrar costes a las familias y a los servicios de AT en el tiempo de desplazamiento y el precio del kilometraje, pero su uso ha de ceñirse al seguimiento de la actividad en un determinado caso o la valoración de una situación puntual. No han de confundirse nunca los ámbitos de la salud y el educativo, entre los que nos movemos

Martín, P. (2021). ¿Atención Temprana a distancia? Experiencia en la covid-19 (marzo-julio 2020) y reflexiones para el futuro. RED Visual: Revista Especializada en Discapacidad Visual, 77, 172-181. https://doi.org/10.53094/ IKKR7253. 
en AT: la eficacia con la eficiencia. La primera siempre hay que buscarla y no se puede cuestionar, mientras que la segunda, al hacer referencia a los recursos utilizados, nunca relegará la equidad (dar a cada uno lo que necesita) y la calidad de vida del menor y su familia.

Tampoco se puede obviar que, para que un aprendizaje sea significativo, ha de ser vivencial, ha de ser «emocionante», algo que solo se puede transmitir cuerpo a cuerpo, y más en el ámbito de la AT.

\section{Referencias bibliográficas}

Federación Estatal de Asociaciones de Profesionales de Atención Temprana (2005). Libro blanco de la Atención Temprana [PDF]. Real Patronato sobre Discapacidad.

Pablo Martín Andrade. Maestro del Equipo de Atención Temprana. Centro de Recursos Educativos de la ONCE en Madrid. Avenida del Doctor García Tapia, 210; 28030 Madrid (España). Correos electrónicos: pmma@once.es y pablomartinandrade@gmail.com. 